\title{
Dynamic quadripolar RadioFrequency and vulvodynia
}

\author{
Francesco De Seta ${ }^{1}$ and Mauro Raichi ${ }^{2 *}$ \\ ${ }^{1}$ University of Trieste and IRCCS B. Garofolo, Trieste, Italy \\ ${ }^{2}$ Clinical Pharmacology and Biophysics Consultant, Milan, Italy
}

\begin{abstract}
Background: Among the several subtypes of vulvodynia or idiopathic vulvar pain lasting for at least 3 months, Localised Provoked Vulvodynia (LPV) is the most highly prevalent clinical variant identified by the "2015 classification". The pathophysiology underlying LPV is still elusive and unclear. The association with recurrent vulvovaginal candidiasis and aerobic vaginitis is most likely prominent in leading to the vestibular nociceptive hypersensitivity that is a distinctive diagnostic marker of LPV. The exploratory non-randomised study herein described was designed to investigate if the demonstrated vulvar remodelling and rejuvenating properties of DQRF (Dynamic Quadripolar RadioFrequency) treatment might be of benefit to control the vestibular pain of LPV. The working hypothesis behind the study was that correcting the mucosal hypotrophy frequent in many LPV women would restore a thriving vestibular and vaginal microorganism ecosystem and break the vicious cycle of recurrent yeast and aerobic infections that leads to exaggerated nociceptive response and LPV hyperesthesia, hyperalgesia, and dyspareunia.

Methods: Prospective cohort of 30 consecutively enrolled premenopausal women with vestibulitis and/or moderate to severe hyperesthesia and pain, dyspareunia or pelvic floor hypercontractility related to recurrent vulvovaginal candidiasis and/or aerobic vaginitis. The first 20 women were treated with four 10 -min DQRF sessions (EVA ${ }^{\mathrm{TM}}$ device) spaced at least 7 to 10 days; the last 10 women, acting as controls, underwent a standard 4-week program of pelvic floor rehabilitation. After the baseline clinical, microbiologic and microscopic assessment, a second follow-up visit was planned no more than 15 days after the fourth (last) DQRF treatment session. Assessed parameters included Lactobacillus and aerobic microflora, polymorphonuclear and clue cells, pH, Nugent score, provoked pain (Swab Test), and severity of vaginal atrophy (Vaginal Health Index).

Results: All women completed the planned four DQRF/EVA ${ }^{\mathrm{TM}}$ or physical therapy sessions without adverse effects. Both treatment strategies significantly reduced the Swab Test provoked pain. The reduction in pain severity seemed to be more marked at the follow-up visit in the DQRF treatment group (mean pain score difference, $-3,55$ ) compared with control women (mean pain score difference, $-3,20$ ), although with only marginal statistical significance ( $\mathrm{p} \approx 0.054$ ). Both treatment strategies improved the vestibular and vaginal environment and mucosal hypotrophy, though more definitely in the DQRF-treated women, as observed for the Vaginal Health Index (DQRF vs. physical therapy pre/post score difference: +5.50 vs. $+5.0, p<0.05)$ and for the microbiologic and microscopic markers of deranged intimate ecology (lactobacilli, Nugent score, polymorphonuclear and clue cells, etc.).

Conclusions: The endoderm-derived vestibule, embriologically distinct from the ectoderm-derived external vulva and the mesoderm-derived vagina, may have a quite peculiar inflammatory and immune reactivity compared with contiguous areas. The outcomes of this preliminary pilot study seem to support the working hypothesis that DQRF-induced subepithelial remodelling in the vestibular areas of hyperesthesia and adjoining mucosa may help to restore the normal Lactobacillusdominated ecology of these areas and to normalise the nociceptive responsiveness of the vestibule. The observed clinical benefits were at least comparable to those of pelvic floor rehabilitation and might have been possibly greater if study cohorts had been larger and statistical power higher in a well-controlled study. Further randomised studies are warranted to validate the working hypothesis and quantitatively estimate the symptomatic benefits and impact on quality of life compared to established vulvodynia therapies.
\end{abstract}

\section{Introduction}

Surprisingly, the origin of the highly prevalent and disabling painful condition that is known as vulvodynia is poorly understood and vulvodynia is still an under-studied and under-diagnosed woman's health issue. Vulvodynia affects up to $28 \%$ of women, often of childbearing age, as estimated in U.S. surveys [1], with an overall direct and indirect pharmacoeconomic burden that has been estimated to rise to 31 to 72 billion dollars per year [2]. According to the new nomenclature of vulvar pain, referred to as the "2015 classification" because agreed on at a consensus conference organised in that year by the International Society for the Study of Vulvovaginal Disease, the International Society for the Study of Women's Sexual Health and the International Pelvic Pain Society, vulvodynia is defined as idiopathic vulvar pain lasting for at least 3 months and is classified into several clinical subtypes (localized, generalized, or mixed; upon contact, spontaneous, or mixed; intermittent or constant; primary or secondary). The "2015 classification" also acknowledges that vulvodynia is unlikely to be a single nosological entity rather than a constellation of symptoms of several disease processes with extensive overlappings [1].

The most common clinical presentation and diagnostic category, Localised Provoked Vulvodynia (LPV) - acute vestibular knife-like or burning pain, or a combination of both, lastingly evoked by even light pressure - has been estimated to affect about $16 \%$ of women over their lifetime; almost $7 \%$ of surveyed women were experiencing unexplained localised pain when interviewed [3]. A recent survey put at $30-48 \%$ the

Correspondence to: Mauro Raichi, Clinical Pharmacology and Biophysics Consultant, Milan, Italy, E-mail: mraichi@gmail.com

Key words: localised provoked vulvodynia, vestibule, hyperalgesia, dynamic quadripolar radiofrequency, recurrent vulvovaginal candidiasis, aerobic vaginitis

Received: April 04, 2018; Accepted: April 21, 2018; Published: April 25, 2018 
women with vulvar burning or pain upon contact lasting for at least 3 months and impacting on sexual activity who never sought care; the same survey put at more than half the women experiencing vestibular pain and looking for a diagnosis who never received it [4].

Though the labia minora, labia majora, the mons pubis, and the perineum are relatively pain-free to pressure and touch, the impact on the woman's sexual life and couple relationship, self-esteem, and mood can be devastating; even such an everyday task as inserting a tampon can be excruciating in severe cases, and dysuria is frequent [5].

Proliferation of vulvar nociceptors and co-morbid conditions, as well as central nervous system, hormonal, myofascial and muscular factors, have all been implicated in the genesis of vulvodynia. Genetic, embryological and congenital factors have also been deemed to be important, possibly with a leading role for inflammation. All these pathophysiological determinants variably contribute in different situations and are interdependent, but the paucity of prospective longitudinal studies still prevents to define a clear flow of causality [2].

Treatment of vulvodynia is at present difficult and poorly codified. The progression of therapeutic strategies in women with vulvodynia is usually consecutive, from non-invasive attempts (psychological support, physical therapy) to drug treatments (e.g., topical hormones, gabapentin, antimycotics and antibiotics) up to surgery, with the latter effective in up to more than $80 \%$ of affected women. However, though complications like pruritus, bleeding, infection, Bartholin duct stenosis and vaginismus are frequent [6]. The whole process is empirically based on trial and error, although algorithms have been proposed based on physical examination findings and laboratory tests [6,7]. The efficacy of psychological interventions, pelvic floor physical therapy, and vestibulectomy for provoked vestibulodynia are quite supported in the international literature; conversely, empirical evidence is still sparse for other treatment options like anti-inflammatory agents, hormones and anticonvulsant medications [8].

The herein discussed non-invasive strategy based on the innovative Dynamic Quadripolar RadioFrequency (DQRF) technology, developed by the Italian biotechnology company Novavision Group S.p.A. (Misinto, Monza-Brianza, Italy) and integrated into the lowenergy DQRF-based EVA ${ }^{\text {tx }}$ device, may be a novel treatment option of vulvodynia.

DQRF is the most recent technology, based on a biophysical approach, aimed at anatomical re-modelling of vulvar tissues and, indirectly, restoration of the vestibular and vaginal ecosystem. The interaction between the reduced flow of energy emitted by the DQRF radio frequency generator and the vestibular subepithelial tissues is biophysically quite peculiar in terms of depth and volume of the target subepithelial vulvar areas and administered energy; the electronically controlled movement and temperature sensors of the EVA ${ }^{\mathrm{m}}$ device (RSS ${ }^{\mathrm{m}}$, Radiofrequency Safety System, technology) allow an easy control of the vestibular subepithelial temperatures [9-11].

The herein presented exploratory non-randomised study in a relatively small sample of LPV women of childbearing age was designed to investigate if the vulvar remodelling properties of the DQRF technology, shown in previous studies, might also be of benefit to control the vestibular LPV pain $[9,10]$. The working hypothesis that guided the study was that remodelling of vestibular and vaginal tissues, beyond its established aesthetic value in women with variable degrees of vulvovaginal hypotrophy [11], could also help to restore a more physiological vestibular and vaginal ecology and the Lactobacillus microflora to its normal role as gatekeeper of the vaginal ecosystem.
This could break the self-sustaining cycle, more extensively discussed in the last section of the paper, of recurrent yeast and aerobic eukaryote infections, dysregulation of vestibular fibroblasts with hyper-expression of proinflammatory cytokines, and nociceptive pain that is thought to be at the core of the most prevalent vulvodynia subtype [12].

\section{Methods}

A prospective cohort of 30 premenopausal women with signs and symptoms of vestibulitis and moderate to severe hyperesthesia and pain, dyspareunia, and/or pelvic floor hypercontractility, was consecutively enrolled in the study between January and October 2017. Candidate women routinely attended the Ambulatory Gynaecology Unit at the University of Trieste as outpatients. Vulvodynia followed Recurrent Vulvovaginal Candidiasis (RVVC) and/or Recurrent Aerobic Vaginitis (RAV) in all women.

To be included in the study, candidate women with vulvodynia should not be undergoing any local or systemic therapy, with special reference to hormone therapies, and should not be pregnant. All women referring a history of atopy or showing evidence of vulvar dystrophy or vulvoperineal unrepaired tears and wounds, acute or chronic vulvar disorders including dermatitis, condylomata and herpes simplex, or considered at high risk for human Papillomavirus infections, were excluded. Any confirmed or unconfirmed suspicion of neurological, endocrinological or dermatologic disorder similarly led to exclusion. All selected women provided written informed consent to anonymous collection of their data before the first DQRF session, and all study materials were peer-reviewed for ethical problems.

The first 20 women consecutively enrolled in the study were treated according to the $\mathrm{EVA}^{\mathrm{m}} / \mathrm{DQRF}$ vulvovaginal treatment protocol illustrated in the text box.

The 1.0-1.3 MHz DQRF generator in the standard $\mathrm{EVA}^{\mathrm{mx}}$ device that was used in the study is equipped with four stainless steel dynamic electrodes on anatomical probes. The maximum emitting power is $55 \mathrm{~W}$, with the four electrodes continuously cycled between receiver and transmitter state. When in the ideal combination, these electrodes convey energy with high precision in the subepithelial layers of the vulva and allow to fine tune the vulvar thermal effect in terms both of tissue volumes and depth. The new technology also eliminates the need for a grounding pad and the need to administer heavy energy burdens with the related risk of burns, as already demonstrated in clinical studies [9-11].

Vulvodynia was associated with RVVC in 15 DQRF-treated women and with RAV in 5 women. The last 10 consecutively enrolled women (controls) underwent 4 weekly sessions of standard pelvic floor rehabilitation.

A baseline assessment before the first DQRF treatment session and a follow-up visit after the end of the treatment program were planned to evaluate the microbiological and phase-contrast ( $x 400$ magnification) microscopic health of the vestibular and vaginal

- Four 10-min sessions, spaced at least 7 to 10 days

- Setting of the radio frequency generator: $1 \mathrm{MHz}$

- Operating power: $8-14 \%$ of the maximum device power $(55 \mathrm{~W})$

- Target temperature in vulvovaginal tissues during procedure: $42^{\circ} \mathrm{C}$ (range $40-43^{\circ} \mathrm{C}$ )

EVA $^{\mathrm{TM}} / \mathrm{DQRF}$ vulvovaginal treatment protocol 
ecosystem. These included Lactobacillus microflora and pH; Nugent score (identification and scoring of large Lactobacillus-like rods, small G. vaginalis-like Gram-positive rods, and curved Mobiluncus spp.-like Gram-variable rods); polymorphonuclear and Gardnerellaspecific "clue cells" in vaginal secretions; diagnostic evidence of partial bacterial vaginosis (no woman with Nugent score $>7$ out of 10 was enrolled); Group-B streptococci, other aerobic bacilli and cocci (e.g., E. coli, S. aureus, S. faecalis), and evidence of aerobic vaginitis. Diagnosis of aerobic vaginitis was based on Donders' scores ( 0 to 10) based on Lactobacillus grade, number of leukocytes, proportion of toxic leukocytes, background flora and proportion of parabasal epitheliocytes, with all parameters attributed partial scores 0 to 2 (slight signs of aerobic vaginitis: summed-up score between 3 and 4; moderate AV: summed-up score between 5 and 6; severe AV: summed-up score between 6 and 10) [13]. Semi-quantitative subjective ordinal scores were applied to microbiological assessments $(0$ to +++$)$. Provoked pain (Swab Test, score 0 to 10) and severity of vaginal atrophy (Vaginal Health Index: vaginal elasticity, fluid volume, $\mathrm{pH}$, epithelial integrity, and moisture scored 1 to 5 ; maximum total score 25) were also assessed at the baseline and the follow-up visits. The second clinical and microbiological follow-up assessment was performed within 2 months of the first DQRF treatment session and no more than 15 days after the fourth DQRF session.

A non-parametric test, the Wilcoxon Signed Rank Test, was applied to the means of the Swab Test pain scores, Nugent scores, $\mathrm{pH}$, and Vaginal Health Index scores of the DQRF-treated and pelvic floor rehabilitation sub-cohorts (follow-up vs. baseline visits). Due to the low numbers involved, outcomes related to the incidence of partial bacterial vaginosis and aerobic vaginitis, as well as microbiologic assays (incidence of lactobacilli, Group-B streptococci, yeasts, etc.) were simply tabulated for qualitative discussion; variations of median values were also qualitatively discussed. Two-sided $95 \%$ confidence levels were used for all statistical tests on means with $\mathrm{p}<0.05$ as cut-off for significance.

\section{Results}

The mean age of enrolled women was 34.0 years in the DQRF group and 33.1 in the pelvic floor rehabilitation group; all women completed the planned four DQRF or physical therapy sessions. No woman of the prospective cohort reported side effects.

Figure 1 illustrates the efficacy of the two treatments as controlling strategies of the provoked pain. The severity of the Swab Test pain significantly improved in both treatment groups over the about 2 months of the study (highly significantly in the DQRF-treated women). The reduction in pain severity at the follow-up visit was more marked in the DQRF treatment group (mean pain score difference, -3,55) compared with control women (mean pain score difference, $-3,20$ ), though the difference approached only marginal statistical significance $(p \approx 0.054)$. The provoked pain median scores improved from 7.0 to 3.5 in the DQRF group, and from 7.0 to 4.0 in the pelvic floor rehabilitation group.

Figure 2 summarises the evolution of the mean Vaginal Health Index scores in the two treatment groups. At baseline assessment, the composite index described a significantly more severe hypotrophy in the DQRF group compared with control women (16.15 vs. 16.60 , respectively; $\mathrm{p}<0.05)$. Conversely, the vestibular and vaginal hypotrophy improved at the follow-up visit to very similar levels in both treatment groups ( 21.65 vs. 21.60 , mean Vaginal Health Index pre/post score difference, $+5,5$ vs. $+5.0, \mathrm{p}<0,05)$. The corresponding

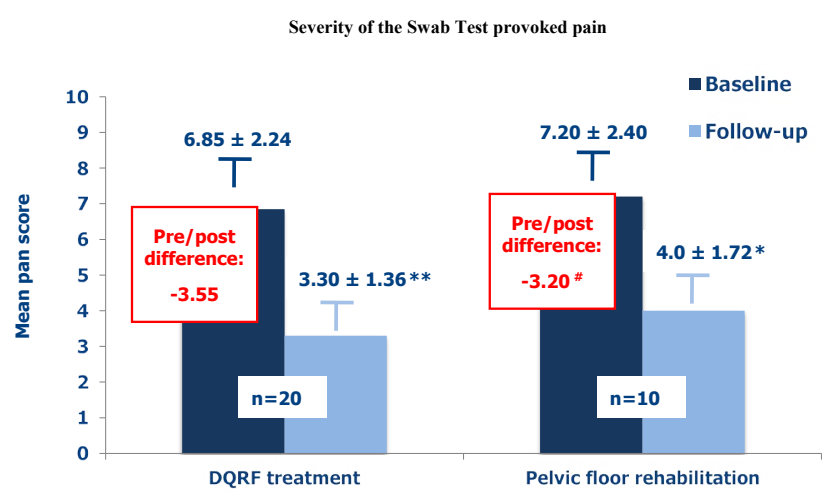

Figure 1. Mean Swab Test provoked pain scores $( \pm$ SEM) before the first DQRF or pelvic floor rehabilitation session (baseline) and at the follow-up visit within 15 days after the end of the 4-session treatment cycle (* $\mathrm{p}<0.05$ vs. baseline visit; ${ }^{* *} \mathrm{p}<0.01$ vs. baseline visit; \# pre- vs. post-difference, $\mathrm{p} \approx 0.054$ ).

Severity of mucosal hypotrophy (Vaginal Health Index)

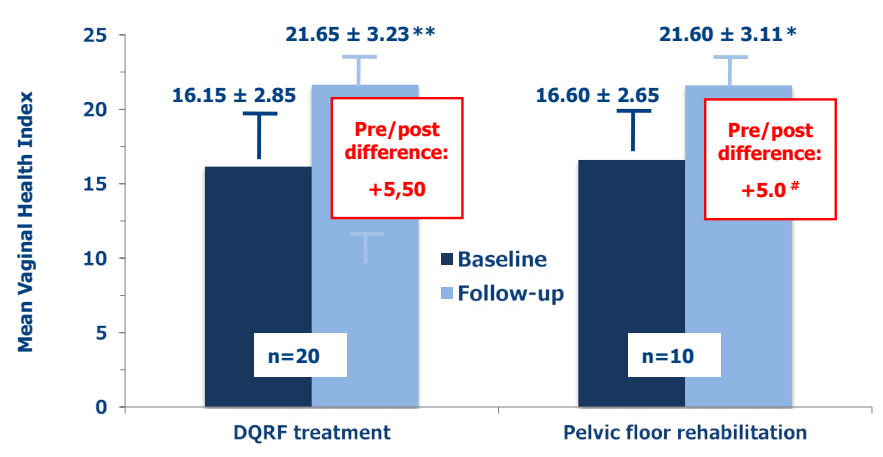

Figure 2. Mean Swab Test provoked pain scores ( \pm SEM) before the first DQRF or pelvic floor rehabilitation session (baseline) and at the follow-up visit within 15 days after the end of the 4-session treatment cycle (* $\mathrm{p}<0.05$ vs. baseline visit; $* * \mathrm{p}<0.01$ vs. baseline visit; \# pre- vs. post-difference, $\mathrm{p}<0.05)$.

median values improved from 16.0 to 21.5 in the DQRF group, and from 16.5 to 21.5 in control women treated with physical therapy.

As regards microbiologic and microscopic evaluations, all five women in the DQRF treatment group that fulfilled the $\mathrm{c}$ lue $\mathrm{c}$ ells criteria for partial bacterial vaginosis at baseline assessment were negative at the follow-up visit; the same was true for women with aerobic vaginitis. Conversely, only one of the five women diagnosed with partial bacterial vaginosis at baseline in the physical therapy group was negative at follow-up. Aerobic vaginitis persisted in both women with that diagnosis at the baseline visit. Group-B streptococci cultures, positive at baseline assessment in the two women of the DQRF group with aerobic vaginitis, were both negative at follow-up, while Group- $B$ streptococci persisted in the two control women with unresolved aerobic vaginitis. The median count of polymorphonuclear cells per optical field in the vaginal swab fell in the DQRF group (from + to 0) but showed no change in the control group (+ at both visits).

Vaginal swab lactobacilli increased at follow-up compared with the baseline visit in the DQRF group (subjective ordinal-score assessment from + to ++ ), but they did not change in the physical therapy group ( + at both visits); the evolution in the mean vestibular and vaginal $\mathrm{pH}$ reflected these changes in the lactobacilli populations (DQRF group: from 4.15 to $3.45, \mathrm{p}<0.05$; control group: from 4.15 to 3.65 , not significant). The median intimate $\mathrm{pH}$ changed from 4.0 to 3.5 in both groups. Table 1 illustrates the descriptive tabulation of the vaginal swab counts of polymorphonuclear cells and lactobacilli per optical field in the treatment groups at the baseline and follow-up visits. 
The observed changes in the Nugent score reflected the pattern of changes in the diagnosis of partial bacterial vaginosis in the two treatment groups (DQRF group: from 3.55 to $2.25, \mathrm{p}<0.001$; control group: from 3.8 to 3.4 , marginally non-significant). The corresponding median values changed from 4.0 to 3.0 in the DQRF group and remained stable at 4.0 in control women (Table 1).

\section{Discussion}

Localised provoked vestibulodynia has long been linked with inflammation, yet failure is almost the rule with treatments aimed at controlling local inflammation.

A careful review of 1,619 studies up to November 2015 failed to support a consistent association between LPV and unambiguous evidence of steady background inflammation [14]. Increased number of mast cells are very frequent in LPV subepithelial areas and activated mast cells produce nerve growth factors and proinflammatory cytokines that are known to induce hyperplasia and sensitisation of the peripheral nociceptive C-fibres. A speculative basis for allodynia might thus be envisioned, yet the authors failed to highlight any steady increase in background proinflammatory cytokines in vestibular tissues of affected women. Conversely, some low degree of inflammatory infiltration of the subepithelial part of the lamina propria is quite normal in healthy women due to the environmental conditions of the vulva, which facilitate proliferation of eukaryotes and yeasts [14]. Steady background inflammation definitely seems a blind alley to explain vulvodynia.

Some clues about LPV pathophysiology are provided by the strong association between vulvodynia and several other pain conditions, e.g., interstitial cystitis, fibromyalgia and irritable bowel syndrome, as well as autoimmune diseases, psoriasis, and atopy $[2,15,16]$. As a consequence, the emphasis has shifted over the last decade to local regulation of immunity and the role of genetics and epigenetics $[12,17,18]$. For instance, natural killer cells are significantly less in LPV women compared with healthy controls [14], in good correlation with the history of previous chronic and recurring Candida infections

Table 1. Vaginal swab polymorphonuclear cells and lactobacilli, counts per optical field; semiquantitative assessments and median scoring at baseline and follow-up visit. Colums 1 and 3: DQRF treatment group $(n=20)$; Colums 2 and 4: pelvic floor rehabilitation group $(n=10)$.

\begin{tabular}{|c|c|c|c|}
\hline \multicolumn{2}{|c|}{$\begin{array}{c}\text { Polymorphonuclear cells (baseline/ } \\
\text { follow-up) }\end{array}$} & \multicolumn{2}{|c|}{ Lactobacilli (baseline/follow-up) } \\
\hline$+/ 0$ & $+++/ 0$ & $+/++$ & $+/++$ \\
\hline$++/+$ & $+/+$ & $+/++$ & $+/+$ \\
\hline$++/++$ & $++/+$ & $+/+$ & $+/+$ \\
\hline$++/ 0$ & $++/+$ & $+/+++$ & $+/+$ \\
\hline $0 / 0$ & $+/+$ & $+/++$ & $+/+$ \\
\hline $0 / 0$ & $++/++$ & $+/+++$ & $+/+$ \\
\hline$+/+$ & $0 / 0$ & $+/+$ & $+/+$ \\
\hline \multirow{2}{*}{$+/ \mathrm{O} 0 / 0$} & $+/+$ & $+/+++$ & $+/+$ \\
\hline & $+/+$ & $++/++$ & $+/+$ \\
\hline $0 / 0$ & $+/+$ & $++/++$ & $+/+$ \\
\hline$+/ 0$ & & $+/+++$ & \\
\hline$+/ 0$ & & $+/+++$ & \\
\hline $0 / 0$ & & $+/++$ & \\
\hline$++/+$ & & $+/++$ & \\
\hline \multirow{2}{*}{ +/O 0/0 } & & $+/+++$ & \\
\hline & & $+/+++$ & \\
\hline$++/+$ & & $+/++$ & \\
\hline $0 / 0$ & & $+/+$ & \\
\hline $0 / 0$ & & $+/+$ & \\
\hline $0 / 0$ & & $+/+$ & \\
\hline Median: +/0 & Median: +/+ & Median: +/++ & Median: $+/+$ \\
\hline
\end{tabular}

(known to occur in up to $70-80 \%$ of LPV women) [5,14]. Toll-like surface receptors 4 , activated in situation of damage or danger and important in the priming of immune cells, are known to be activated by Candida infections, further reinforcing the correlation between recurrent candidiasis and vestibulodynia without obvious baseline inflammation [19-21]. Similarly, activation of the immune system can probably be documented in up to three quarters of LPV women, with most instances of abundant mast cell infiltration being in fact a secondary mast cell disorder [14]. The role of immune activation in provoked vestibulodynia is further supported by the association between epithelial nerve hyperplasia and increased B-cell infiltration and germinal centres [22].

These observations could explain the correlation between bacterial vaginosis and trichosomiasis and vestibular pain that has also been documented $[23,24]$, possibly even more strongly than the association with recurrent candidiasis (according to Smith et al, the odds ratio is 9.4 for physician-reported bacterial vaginosis vs. 5.7 for Candida infections and 20.6 for trichosomiasis) [23].

Yet more evidences: although there may be little baseline inflammation, the vestibular fibroblasts from LPV women express more proinflammatory cytokines such as IL-1ß, IL-6 and IL-8 compared with healthy women after in-vitro stimulation $[12,14,18]$; likewise, inflammation-triggered control mechanisms, e.g., expression of the IL-1 receptor antagonist, appear genetically less efficient in LPV women [14]. Vestibular fibroblasts from painful areas of LPV women also strongly express Dectin-1, a transmembrane pattern-recognition receptor with high binding affinity for the $C$. albicans cell wall glucan and an important role in anti-fungal innate immunity [5,17]. Dectin-1 expression in areas of hyperalgesia is significantly higher compared with expression from fibroblasts from non-painful external vulvar areas at a short distance: blocking the function or expression of Dectin-1 e.g., via the NFKB pathway - is associated with a reduced expression of inflammatory cytokines. Inhibition of the NFkB pathway has already been targeted clinically, almost eliminating proinflammatory mediator secretion in vulvar fibroblasts $[5,17,25]$. Finally, the very short endoderm-derived vestibule, interposed between the ectoderm of the external vulva and the vaginal mesoderm, is embriologically, and possibly functionally, quite peculiar. A tendency to local cytokine dysregulation and ineffective control of induced inflammation might contribute to local nociceptor sensitisation in vulvodynia-afflicted women $[12,18]$.

Summarising, a growing body of evidence seems to suggest a mechanistic connection among the described actors: proinflammatory stimulants triggering fibroblast activation in the vestibular region even with low or nil baseline inflammation, peculiarly responsive fibroblasts, enhanced expression of proinflammatory cytokines, and nociceptive pain. In the words of Foster et al, vulvodynia should not even be considered a real disorder, rather than "an extreme but natural phenomenon" in an embriologically unique tiny area, the inch or so of the vulvar vestibule, extensively exposed to trauma and foreign proteins during reproduction and thus prone to "unique inflammatory/ immunologic responsiveness" [12].

Neurobiological factors are also quite likely to influence vulvodynia. Generalized hyperalgesia, meaning lower local and remote pain thresholds (i.e., after vulvar, and after thumb deltoid and shin pressure, respectively) is definitely a factor in vulvodynia. This is well highlighted by functional magnetic resonance imaging (fMRI) during vulvar and thumb stimulation, which shows enhanced brain activation within the insula, the thalamus and the dorsal mid-cingulate and posterior 
cingulate cortex of LPV women compared with age-matched pain-free controls. Neural fMRI activation correlates with pain severity; different levels of neural activation are also specific of the recognised vulvodynia subgroups, meaning primary versus secondary and provoked versus unprovoked subgroups, as further hint that several heterogeneous disorders actually coexist under the label of vulvodynia [26]. However, it is still unclear whether the increased central pain processing and generalised hyperalgesia, exemplified by the pain stimulated at sites remote from the vulva like the thumb, are secondary to a primary vestibular disorder of pain control, or whether the augmented neural activity is in fact driving the perceived pain. Few are the accepted facts: for instance, that remission of vulvodynia symptoms is frequent, but relapses are likewise common whilst persistence without remission is quite exceptional rather than the rule [27].

In this far from clear pathophysiological background, this exploratory study supports the working hypothesis that guided its design: the vestibular and vaginal microflora ecology improves as a consequence of remodelling and rejuvenation of the vulvovaginal mucosa. Most likely, this helps to break the self-sustaining flow of events that leads to the enhanced inflammatory responsiveness of the LPV vestibule, even with no or minimal evidence of background inflammation, and excruciating nociceptive pain [12]. The recently developed DQRF/EVA ${ }^{\text {ma }}$ technology has shown a peculiarly strong remodelling efficacy of the vulvovaginal anatomy in a variety of conditions [9-11]. This novel radiofrequency technology was thus chosen to test the working hypothesis in comparison with pelvic floor physical therapy, which is known to be of benefit in most LPV patients [28].

Only in 2013 did the first report appear of successful use of pulsed radiofrequency in the treatment of severe refractory (neuropathic) vulvodynia-i.e., nosologically distinct from LPV according to the "2015 classification" $[2,29]$. Since then, pulsed radiofrequency has been widely used with no clear rationale in this frankly neuropathic indication. Another 2016 pilot study described the efficacy of fractional $\mathrm{CO}_{2}$ laser treatment of the vestibule in women with idiopathic vulvodynia and vestibular pain associated with the genitourinary syndrome of menopause. Some benefits were observed - possibly because, in the vulva and vagina, the loss of oestrogens is associated with increased density of sensory nerve fibres per unit area - yet once again not in LPV [30]; without forgetting that painful scars and vulvodynia may follow $\mathrm{CO}_{2}$ laser treatment [31]. As far as we know, this may well be the first study applying a radiofrequency technology to the treatment of LPV.

Both the DQRF/ EVA ${ }^{\mathrm{si}}$ device and the standard pelvic physical therapy both appeared to control the Swab Test provoked pain and to improve the mucosal hypotrophy. The outcomes of the study seem to suggest that the DQRF/ EVA ${ }^{\text {mix }}$ technology could have an overall higher efficacy, yet the data cannot suggest at the moment anything more than a tendency due to the non-randomised design of the study. A non-randomised design is justified in an exploratory study, but further randomised evidences are warranted. However, there is at least a revealing clue, the microbiologic changes of the vestibular and vaginal microflora, which might suggest a significant efficacy of the new radiofrequency technology. The DQRF technology was definitely more efficient that physical therapy in restoring a healthy Lactobacillus population and in suppressing the polymorphonuclear and clue cells infiltration. That goes hand in hand with its high efficacy in almost normalising the Vaginal Health Index though starting from a situation of comparatively worse mucosal hypotrophy (final mean score in DQRF-treated women - 21.65 out of a maximum of 25).
The effect on pain was most unlikely to be a placebo effect. A most recent small metanalysis of topical medications as monotherapy of vulvodynia showed placebo to be as effective as any medication [32]. This does not seem the case for the DQRF/ EVA ${ }^{\mathrm{mw}}$ technology. First of all, the study was limited to women with localised provoked vulvodynia and did not include women with the less frequent idiopathic clinical subtype, which as a neuropathic disorder is liable to placebo effects. Secondly, reduction of pain with the DQRF physical treatment was associated with a shift towards normality of the depleted Lactobacillus vestibular microflora as well as other signs of ecological and microscopic improvement. Such improvements seem unlikely to be a mere placebo effect.

In conclusion, the outcomes of this ground breaking exploratory study suggest that the new DQRF technology might be a promising new strategy to control provoked vulvodynia and the severe impact on female self-esteem that is all too often associated with this poorly recognised disorder. Any final judgement must wait for new soundly designed trials.

\section{Conflicts of Interest}

The authors were in the past consultants to Novavision Group S.p.A. They certify to have no current conflict of interest with any financial or commercial organization regarding the content of this manuscript.

\section{References}

1. Groysman V (2010) Vulvodynia: new concepts and review of the literature. Dermatol Clin 28: 681-696. [Crossref]

2. Pukall CF, Goldstein AT, Bergeron S, Foster D, Stein A, et al. (2016) Vulvodynia Definition, Prevalence, Impact, and Pathophysiological Factors. J Sex Med 13: 291304. [Crossref]

3. Harlow BL, Stewart EG (2003) A population-based assessment of chronic unexplained vulvar pain: have we underestimated the prevalence of vulvodynia? J Am Med Womens Assoc 58: 82-88. [Crossref]

4. Harlow BL, Kunitz CG, Nguyen RH, Rydell SA, Turner RM, et al. (2014) Prevalence of symptoms consistent with a diagnosis of vulvodynia: population-based estimates from 2 geographic regions. Am J Obstet Gynecol 210: 40. e1-e8. [Crossref]

5. Falsetta ML, Foster DC, Woeller CF, Pollock SJ, Bonham AD, et al. (2015) Identification of novel mechanisms involved in generating localized vulvodynia pain. Am J Obstet Gynecol 213: 38.e1-e12. [Crossref]

6. Landry T, Bergeron S, Dupuis MJ, Desrochers G (2008) The treatment of provoked vestibulodynia: a critical review. Clin J Pain 24: 155-171. [Crossref]

7. King M, Rubin R, Goldstein AT (2014) Current uses of surgery in the treatment of genital pain. Curr Sex Health Rep 6: 252-258.

8. Goldstein AT, Pukall CF, Brown C, Bergeron S, Stein A, et al. (2016) Vulvodynia: Assessment and Treatment. J Sex Med 13: 572-590. [Crossref]

9. Vicariotto F, Raichi M (2016) Technological evolution in the radiofrequency treatment of vaginal laxity and menopausal vulvovaginal atrophy and other genitourinary symptoms: first experiences with a novel dynamic quadripolar device. Minerva Ginecol 68: 225-236. [Crossref]

10. Vicariotto F, DE Seta F, Faoro V, Raichi M (2017) Dynamic quadripolar radiofrequency treatment of vaginal laxity/menopausal vulvo-vaginal atrophy: 12-month efficacy and safety. Minerva Ginecol 69: 342-349. [Crossref]

11. Benincà G, Bosoni D, Vicariotto F, Raichi M (2017) Efficacy and safety of Dynamic Quadripolar Radio-Frequency, a new high-tech, high-safety option for vulvar rejuvenation. Obstet Gynecol Rep 1: 1-5.

12. Foster DC, Piekarz KH, Murant T, LaPoint R, Haidaris CG, et al. (2007) Enhanced synthesis of proinflammatory cytokines by vulvar vestibular fibroblasts: implications for vulvar vestibulitis. Am J Obstet Gynecol 196: 346.e1-8. [Crossref]

13. Donders GG (2007) Definition and classification of abnormal vaginal flora. Best Pract Res Clin Obstet Gynaecol 21: 355-73 [Crossref] 
14. Chalmers KJ, Madden VJ, Hutchinson MR, Moseley GL (2016) Local and systemic inflammation in localized, provoked vestibulodynia: a systematic review. Obstet Gynecol 128: 337-347. [Crossref]

15. Regauer S, Eberz B, Beham-Schmid C (2015) Mast cell infiltrates in vulvodynia represent secondary and idiopathic mast cell hyperplasias. APMIS 123: 452-456. [Crossref]

16. Fariello JY, Moldwin RM (2015) Similarities between interstitial cystitis/bladder pain syndrome and vulvodynia: implications for patient management. Transl Androl Urol 4: 643-652. [Crossref]

17. Lev-Sagie A, Witkin SS (2016) Recent advances in understanding provoked vestibulodynia. F1000Res 5: 2581. [Crossref]

18. Babula O, Linhares IM, Bongiovanni AM, Ledger WJ, Witkin SS (2008) Association between primary vulvar vestibulitis syndrome, defective induction of tumor necrosis factor-alpha, and carriage of the mannose-binding lectin codon 54 gene polymorphism. Am J Obstet Gynecol 198: 101.e1-4. [Crossref]

19. Netea MG, Gow NA, Munro CA, Bates S, Collins C, et al. (2006) Immune sensing of Candida albicans requires cooperative recognition of mannans and glucans by lectin and Toll-like receptors. J Clin Invest 116: 1642-1650. [Crossref]

20. Gil ML, Gozalbo D (2009) Role of Toll-like receptors in systemic Candida albicans infections. Front Biosci (Landmark Ed) 14: 570-582. [Crossref]

21. Falsetta ML, Foster DC, Woeller CF, Pollock SJ, Bonham AD, et al. (2018) Toll-Like receptor signaling contributes to proinflammatory mediator production in localized provoked vulvodynia. J Low Genit Tract Dis 22: 52-57. [Crossref]

22. Tommola P, Unkila-Kallio L, Paetau A, Meri S, Kalso E, et al. (2016) Immune activation enhances epithelial nerve growth in provoked vestibulodynia. Am J Obstet Gynecol 215: 768.e1-768.e8. [Crossref]
23. Smith EM, Ritchie JM, Galask R, Pugh EE, Jia J, et al. (2002) Case-control study of vulvar vestibulitis risk associated with genital infections. Infect Dis Obstet Gynecol 10: 193-202 [Crossref]

24. Edgardh K, Abdelnoor M (2007) Vulvar vestibulitis and risk factors: a populationbased case-control study in Oslo. Acta Derm Venereol 87: 350-354. [Crossref]

25. Falsetta ML, Foster DC, Bonham AD, Phipps RP (2017) A review of the available clinical therapies for vulvodynia management and new data implicating proinflammatory mediators in pain elicitation. BJOG 124: 210-218. [Crossref]

26. Hampson JP, Reed BD, Clauw DJ, Bhavsar R, Gracely RH, et al. (2013) Augmented central pain processing in vulvodynia. J Pain 14: 579-589. [Crossref]

27. Reed BD, Harlow SD, Plegue MA, Sen A (2016) Remission, relapse, and persistence of vulvodynia: a longitudinal population-based study. $J$ Womens Health (Larchmt) 25: 276-283. [Crossref]

28. Edwards L (2015) Vulvodynia. Clin Obstet Gynecol 58: 143-152. [Crossref]

29. Kestranek J, Spacek J, Ryska P, Adamkov J, Matula V, et al. (2013) Radiofrequency therapy for severe idiopathic vulvodynia. J Low Genit Tract Dis 17: e1-4. [Crossref]

30. Murina F, Karram M, Salvatore S, Felice R (2016) Fractional CO2 laser treatment of the vestibule for patients with vestibulodynia and genitourinary syndrome of menopause: a pilot study. J Sex Med 13: 1915-1917. [Crossref]

31. Tschanz C, Salomon D, Skaria A, Masouye I, Vecchietti GL, et al. (2001) Vulvodynia after CO2 laser treatment of the female genital mucosa. Dermatology 202: 371-372. [Crossref]

32. Varella Pereira GM, Marcolino MS, Nogueira Reis ZS, de Castro Monteiro MV (2018) A systematic review of drug treatment of vulvodynia: evidence of a strong placebo effect. BJOG. [Crossref]

Copyright: C2018 Seta FD. This is an open-access article distributed under the terms of the Creative Commons Attribution License, which permits unrestricted use, distribution, and reproduction in any medium, provided the original author and source are credited. 